\title{
Line identification in soft X-ray spectra of stellar coronae by comparison with the hottest white dwarf's photosphere: Procyon, $\alpha$ Cen A+B, and H 1504+65
}

\author{
K. Werner ${ }^{1}$ and J. J. Drake ${ }^{2}$ \\ ${ }^{1}$ Institut für Astronomie und Astrophysik, Universität Tübingen, Sand 1, 72076 Tübingen, Germany \\ e-mail: werner@astro.uni-tuebingen.de \\ ${ }^{2}$ Harvard-Smithsonian Center for Astrophysics, MS 3, 60 Garden Street, Cambridge, MA 02138, USA
}

Received 23 September 2004 / Accepted 8 January 2005

\begin{abstract}
H 1504+65 is a young white dwarf with an effective temperature of $200000 \mathrm{~K}$ and is the hottest post-AGB star ever analysed with detailed model atmospheres. Chandra LETG+HRC-S spectra have revealed the richest X-ray absorption line spectrum recorded from a stellar photosphere to date. The line forming regions in this extremely hot photosphere produce many transitions in absorption that are also observed in emission in cool star coronae. We have performed a detailed comparison of Chandra spectra of H 1504+65 with those of Procyon and $\alpha$ Cen A and B. State of the art non-LTE model spectra for the hot white dwarf have enabled us to identify a wealth of absorption lines from highly ionized $\mathrm{O}, \mathrm{Ne}$ and $\mathrm{Mg}$. In turn, these features have allowed us to identify coronal lines whose origins were hitherto unknown.
\end{abstract}

Key words. stars: atmospheres - stars: coronae - X-rays: stars - stars: individual H 1504+65

\section{Introduction}

High-resolution X-ray spectroscopy performed with Chandra and XMM-Newton allows very detailed studies of coronae about cool stars. While many individual emission lines were detected for the first time in stellar spectra by the Extreme Ultraviolet Explorer Satellite (EUVE; see, e.g., Drake et al. 1995), the resolving power of $\lambda / \Delta \lambda \sim 200$ of the EUVE spectrographs was quite modest compared with that of present day $\mathrm{X}$-ray observatories. In particular, the unprecedented resolution capabilities of the Chandra X-ray Observatory Low Energy Transmission Grating Spectrograph (LETG) in the 30-170 $\AA$ range $(\lambda / \Delta \lambda \sim 1000)$ that overlaps with the EUVE Short Wavelength spectrometer (70-170 ̊), have revealed many more weak spectral lines.

The 25-70 $\AA$ region is a relatively uncharted part of the soft X-ray spectrum. Prior to Chandra, only a small handful of astrophysical observations had been made at anything approaching high spectral resolution in this range: these were of the solar corona using photographic spectrometers (Widing \& Sandlin 1968; Freeman \& Jones 1970; Schweizer \& Schmidtke 1971; Behring et al. 1972; Acton et al. 1985) a channel electron photomultiplier (Malinovsky \& Heroux 1973) and a GeigerMüller counter (Manson 1972). While these works resulted in identifications for many of the bright spectral lines, a large fraction of the forest of weaker features remains unidentified.
Identification of these features is desirable because they could be used as spectroscopic diagnostics, because they potentially contribute to the flux of diagnostic lines currently employed, and because they contribute to the overall plasma radiative loss.

Two nearby stars that have illuminated the forest of lines in the 30-170 $\AA$ range are $\alpha$ Cen $(\mathrm{G} 2 \mathrm{~V}+\mathrm{K} 1 \mathrm{~V})$ and Procyon (F5IV). All three stars exhibit classical solar-like X-ray emitting coronae. Indeed, analogues of the relatively X-ray faint Sun are difficult to observe because they become unreachable with current instrumentation beyond a few parsecs, and $\alpha$ Cen and Procyon represent the nearest and brightest coronal sources with solar-like activity. Only a small fraction of the multitude of lines between $30-170 \AA$ seen in their Chandra LETG spectra could be identified based on current radiative loss models (Raassen et al. 2002, 2003). Drake et al. (in prep.) have estimated that these models underestimate the true line flux in the range $30-70 \AA$ in these stars by factors of up to 5 or so.

The "missing lines" are predominantly transitions involving $n=2$ ground states in abundant elements such as $\mathrm{Ne}$, $\mathrm{Mg}, \mathrm{Si}, \mathrm{S}$ and $\mathrm{Ar}-$ the analogous transitions to the $\mathrm{Fe}$ " $\mathrm{L}$ shell" lines between $\sim 8-18 \AA$, together with Fe $n=3$ (the "M-shell") transitions (Drake 1996; Drake et al. 1997; Jordan 1996). Some of these lines have been identified based on Electron Beam Ion Trap experiments (Beiersdorfer et al. 1999; Lepson et al. 2002, 2003). In the present paper we approach this problem from a new perspective, namely through a Chandra 
observation of the photosphere of the hottest white dwarf (WD) known, H 1504+65, and its quantitative analysis by means of detailed non-LTE model atmospheres.

H 1504+65 has an effective temperature of $200000 \mathrm{~K}$. It belongs to the PG1159 spectral class, which are hot, hydrogendeficient (pre-) white dwarfs. Their surface chemistry (typical abundances: $\mathrm{He}=33 \%, \mathrm{C}=48 \%, \mathrm{O}=17 \%, \mathrm{Ne}=2 \%$, mass fractions) suggests that they exhibit matter from the heliumbuffer layer between the $\mathrm{H}$ - and He-burning shells in the progenitor AGB star (Werner 2001). This is likely because the PG1159 stars have suffered a late He-shell flash, a phenomenon that drives the fast evolutionary rates of such famous stars like FG Sge and Sakurai's object. H 1504+65 is in fact a peculiar member of this class, because it is also helium-deficient. Its atmosphere is mainly composed of carbon and oxygen plus neon and magnesium $(\mathrm{C}=48 \%, \mathrm{O}=48 \%, \mathrm{Ne}=2 \%, \mathrm{Mg}=2 \%$, mass fractions). $\mathrm{H} 1504+65$ is a unique object, considering its high $T_{\text {eff }}$ and chemical surface composition, and we have speculated that it represents the naked $\mathrm{C} / \mathrm{O}$ core of a former red giant (Werner et al. 2004, W04).

Chandra $\mathrm{LETG}+\mathrm{HRC}-\mathrm{S}$ spectra from $\mathrm{H}$ 1504+65 have revealed the richest X-ray absorption line spectrum recorded from a stellar photosphere to date. We have recently performed a detailed analysis of this spectrum (W04) and we use in the paper in hand the photospheric spectrum of $\mathrm{H} \mathrm{1504+65}$ together with an appropriate model atmosphere to identify a number of emission lines in the coronae of $\alpha \operatorname{Cen} \mathrm{A}, \alpha$ Cen B, and Procyon. The difference in particle densities in the WD photosphere and in the coronae amounts to many orders of magnitude (roughly $n_{\mathrm{e}}=10^{13}-10^{18}$ and $10^{10} \mathrm{~cm}^{-3}$, respectively), however, the temperature in the line forming regions of the WD (up to $300000 \mathrm{~K}$ ) is comparable to the low-temperature component of multi-temperature fits to coronae, required to account for the lines of low-ionization stages (e.g. $630000 \mathrm{~K}$ for Procyon; Raassen et al. 2002). As a consequence, numerous lines from O VI, Ne VI-VIII and Mg VI-IX are visible in the soft X-ray spectra of both, the cool star coronae (in emission) and the hot WD photosphere (in absorption). Lines from higher ionization stages are formed in the high-temperature regions of the coronae ( $\mathrm{T}$ of the order $1-2.5$ million $\mathrm{K}$ for the stars studied in this paper), hence, their respective absorption line counterparts cannot be formed in the WD photosphere.

In the following, we first introduce briefly the characteristics of the objects studied here. We describe our model atmosphere calculation for the hot WD, concentrating on the atomic data employed. We then perform a detailed comparison of the absorption and emission line spectra and suggest a number of new line identifications for the cool star coronae.

\section{Observations}

H 1504+65 was observed with the Chandra LETG+HRC-S on September 27, 2000, with an integration time of approximately $25 \mathrm{ks}$. Flux was detected in the range $60 \AA-160 \AA$. The spectrum is that of a hot photosphere, characterized by a continuum with a large number of absorption lines from highly ionized species: O V-VI, Ne VI-VIII, and Mg V-VIII. It rolls off at long wavelengths due to ISM absorption. The maximum flux is detected near $110 \AA$. Between $105 \AA$ and $100 \AA$ the flux drops because of photospheric absorption from the O VI edge caused by the first excited atomic level. The edge is not sharp because of a converging line series and pressure ionization. Below $100 \AA$ the flux decreases, representing the Wien tail of the photospheric flux distribution. The complete spectrum with detailed line identifications was presented in W04.

The $\alpha$ Cen A and B observation has been described in detail by Raassen et al. (2002) and we describe it here only in brief. $\alpha$ Cen was observed with the LETG + HRC-S on December 25, 1999 with an exposure time of $81.5 \mathrm{ks}$, including dead time corrections to account for telemetry saturation during intervals of high background. The observation was designed such that the two stars were maximally separated in the cross-dispersion axis, with the dispersion axis positioned nearly perpendicular to the axis of the binary. At the time of the observation, the stars were separated by $16^{\prime \prime}$ on the sky. The spectra were extracted with the standard CIAO bow-tie region, though the central two background regions interfered with the stellar spectra and only the outer regions were used for background subtraction.

The two Procyon observations studied here were obtained with the LETG+HRC-S as part of the Chandra on-orbit calibration programme and Emission Line Project. The observations were executed contiguously beginning on November 6 , 1999 at 21:11:32 UT. The second observation began on 1999 November 16:59:48 UT. The effective exposure times for these observations were $69643 \mathrm{~s}$ and $69729 \mathrm{~s}$, respectively, including dead time corrections.

Reduction of the HRC-S event lists for all the observations was initially based on standard pipeline products. Events were further filtered in pulse height in order to remove background events. The final reduced first order spectra were co-added in order to maximise the signal. In the case of Procyon, we also co-added the two separate observations.

\section{Photospheric model for $\mathrm{H} 1504+65$}

We use here a photospheric spectrum from a line blanketed non-LTE model atmosphere constructed for $\mathrm{H} 1504+65$ by W04. Model parameters are: $T_{\text {eff }}=200000 \mathrm{~K}, \log g=8$ $\left[\mathrm{cm} \mathrm{s}^{-2}\right]$, and $\mathrm{C}=48 \%, \mathrm{O}=48 \%, \mathrm{Ne}=2 \%, \mathrm{Mg}=2 \%$, (mass fractions). Details of model assumptions and calculations can be found in that reference and we restrict ourselves here to those characteristics which are of immediate relevance in our context. This primarily concerns the NLTE model atoms for neon and magnesium. They comprise 88 and 122 NLTE levels, connected with 312 and 310 radiative line transitions, respectively, in the ionization stages IV-IX. The final synthetic spectrum was computed considering fine structure splitting of levels and multiplets assuming relative LTE populations for levels within a particular term. We have tried to use the best available data for level energies and line wavelengths, compiling them from several sources. For the lines discussed here (Table 1), we used the following databases:

(i) National Institute of Standards and Technology (NIST) ${ }^{1}$;

\footnotetext{
${ }^{1}$ http://physics.nist.gov/
} 
Table 1. List of X-ray multiplets in the wavelength region 69-151 Å observed in both the $\mathrm{H} 1504+65$ photosphere or its model and in the coronae of either $\alpha$ Cen A ("A"), $\alpha$ Cen B ("B"), or Procyon ("P"), as suggested in this paper. In the last column, we note earlier line identifications in either solar spectra (SA = Acton et al. 1985; SB = Behring et al. 1972; SF = Freeman \& Jones 1970; SM = Manson 1972; SMH = Malinovsky \& Heroux 1973; SW = Widing \& Sandlin 1968) or stellar spectra (D = Drake et al. 1995; $R=$ Raassen et al. 2002, 2003). The letter "u" is appended in the case of the feature having been observed but not identified. "N" denotes a new identification suggested in this paper. "N" in combination with other letters means that at least one component of the multiplet is newly identified here. Expressions in brackets denote doubtful cases. The column "Source" gives the reference to the level energies of the transition. After each transition we have marked, if the lower level is a ground state ("G") or a metastable state ("M").

\begin{tabular}{|c|c|c|c|c|c|c|}
\hline$\lambda / \AA ̊($ H $1504+65$ model $)$ & Seen in & Ion & Transition & & Source & Remark \\
\hline $69.41, .47, .57$ & $\mathrm{~A}, \mathrm{~B}, \mathrm{P}$ & Mg VIII & $\begin{array}{c}2 \mathrm{p}^{2} \mathrm{P}^{\mathrm{o}}-3 \mathrm{p}{ }^{2} \mathrm{D} \\
-\end{array}$ & G & Nist & $\begin{array}{l}\text { N, SMu, blend with blue wing of } \\
\text { Si VIII } 69.63 \AA\end{array}$ \\
\hline $74.27, .32, .34, .37, .41, .43$ & $\mathrm{~A}, \mathrm{~B}, \mathrm{P}$ & Mg VIII & $2 \mathrm{p}^{24} \mathrm{P}-3 \mathrm{~d}{ }^{4} \mathrm{D}^{\mathrm{o}}$ & M & Nist & $\mathrm{N}, \mathrm{SAu}, \mathrm{SMu}$, broad emission feature \\
\hline $74.78, .81, .87$ & $\mathrm{~A}, \mathrm{~B}$ & $\mathrm{Ne}$ VII & $2 p^{3} P^{o}-4 p^{3} D$ & M & NisT & N, SAu, SBu, SMu, blend with Mg VIII $74.86 \AA$ \\
\hline $74.86,75.03, .04$ & $\mathrm{~A}, \mathrm{~B}, \mathrm{P}$ & Mg VIII & $\begin{array}{c}2 \mathrm{p}^{2} \mathrm{P}^{\mathrm{o}}-3 \mathrm{~d}{ }^{2} \mathrm{D} \\
-\end{array}$ & G & Nist & $\begin{array}{l}\text { SA, SB, SF, SM, SMH, SW, R, blend with } \\
\text { Fe XIII } 74.85 \text { noted by R, and Ne VII } 74.87 \AA\end{array}$ \\
\hline (78.34), $78.41,78.52$ & $\mathrm{~A}, \mathrm{~B}, \mathrm{P}$ & Mg VII & $2 \mathrm{p}^{3}{ }^{3} \mathrm{P}-3 \mathrm{p}{ }^{3} \mathrm{P}^{\mathrm{o}}$ & G & Kelly & $\mathrm{N}, \mathrm{SAu}, \mathrm{SMu}$ \\
\hline $80.23, .25$ & $\mathrm{~A}, \mathrm{~B}, \mathrm{P}$ & Mg VIII & $2 \mathrm{p}^{2} \mathrm{D}-3 \mathrm{~d}{ }^{2} \mathrm{D}^{\mathrm{o}}$ & & Nist & $\mathrm{SMu}, \mathrm{R}$ \\
\hline $80.95,81.02, .14$ & $\mathrm{~A},(\mathrm{~B}, \mathrm{P})$ & Mg VII & $2 \mathrm{p}^{3}{ }^{3} \mathrm{P}-3 \mathrm{p}{ }^{3} \mathrm{~S}^{o}$ & $\mathrm{G}$ & Kelly & $\mathrm{N}, \mathrm{SAu}, \mathrm{SMu}$ \\
\hline 81.37 & (A), B, P & $\mathrm{Ne}$ VII & $2 p^{1} \mathrm{P}^{\mathrm{o}}-4 \mathrm{p}^{1} \mathrm{P}$ & & Kelly & $\mathrm{N}$ \\
\hline $81.73, .79, .84, .87, .94, .98$ & $\mathrm{~A}, \mathrm{~B}, \mathrm{P}$ & Mg VIII & $\begin{array}{c}2 \mathrm{p}^{2}{ }^{4} \mathrm{P}-3 \mathrm{~s}{ }^{4} \mathrm{P}^{\mathrm{o}} \\
-\end{array}$ & M & Nist & $\begin{array}{l}\text { N, SFu, SMu, broad emission, blend with } \\
\text { Si VII } 81.89 \AA \text { A noted by R }\end{array}$ \\
\hline $82.17, .20, .27$ & $\mathrm{~A}, \mathrm{~B},(\mathrm{P})$ & $\mathrm{Ne}$ VII & $2 p{ }^{3} \mathrm{P}^{\mathrm{o}}-4 \mathrm{~d}^{3} \mathrm{D}$ & M & Kelly & $\mathrm{N}$ \\
\hline (82.60), .82 & $\mathrm{A}, \mathrm{B}, \mathrm{P}$ & Mg VIII & $\begin{array}{c}2 \mathrm{p}^{2} \mathrm{P}^{\mathrm{o}}-3 \mathrm{~s}{ }^{2} \mathrm{~S} \\
-\end{array}$ & G & Nist & $\begin{array}{l}(\mathrm{SBu}, \mathrm{SFu}), \mathrm{SM}, \mathrm{R} \text {, blend with Fe XII } 82.84 \AA \\
\text { noted by } \mathrm{R}\end{array}$ \\
\hline $83.51, .56, .59, .64, .71, .76$ & $\mathrm{~A}, \mathrm{~B}, \mathrm{P}$ & Mg VII & $\begin{array}{c}2 \mathrm{p}^{33} \mathrm{P}-3 \mathrm{~d}{ }^{3} \mathrm{P}^{\mathrm{o}} \\
-\end{array}$ & $\mathrm{G}$ & Kelly & $\begin{array}{l}\text { N, SBu, SF, SW, SM, D, R, broad emission } \\
\text { feature; poss. Si VI contribution noted by R }\end{array}$ \\
\hline $83.91, .96, .99,84.02, .09, .11$ & $\mathrm{~A}, \mathrm{~B}, \mathrm{P}$ & Mg VII & $\begin{array}{c}2 \mathrm{p}^{33} \mathrm{P}-3 \mathrm{~d}{ }^{3} \mathrm{D}^{\mathrm{o}} \\
-\end{array}$ & $\mathrm{G}$ & Kelly & $\begin{array}{l}\mathrm{N}, \mathrm{SB}, \mathrm{SF}, \mathrm{SM}, \mathrm{SMH}, \mathrm{R} \text {, broad } \\
\text { emission feature }\end{array}$ \\
\hline$(84.19, .23) .30$, & A & $\mathrm{Ne}$ VII & $2 p^{3} \mathrm{P}^{\mathrm{o}}-4 \mathrm{~s}^{3} \mathrm{~S}$ & M & Bashkin & $\mathrm{SMu}, \mathrm{R}$ \\
\hline 85.41 & $(\mathrm{~A}, \mathrm{~B}, \mathrm{P})$ & Mg VII & $\begin{array}{c}2 \mathrm{p}^{2}{ }^{1} \mathrm{D}-3 \mathrm{~d}{ }^{1} \mathrm{~F}^{\mathrm{o}} \\
-\end{array}$ & M & Kelly & $\begin{array}{l}\text { N, SBu, SMu, blend with Fe XII } 85.46 \AA \\
\text { noted by R }\end{array}$ \\
\hline 86.82 & $\mathrm{~A}, \mathrm{~B}, \mathrm{P}$ & Ne VII & $\begin{array}{c}2 \mathrm{p}^{2}{ }^{1} \mathrm{D}-4 \mathrm{~d}^{1} \mathrm{~F}^{\mathrm{o}} \\
-\end{array}$ & M & Kelly & $\begin{array}{l}\text { N, SBu, SFu, SMu, blend with Fe XI } 86.77 \text {, } \\
\text { Mg VIII } 86.84 \text { A noted by R }\end{array}$ \\
\hline $86.84, .85,87.02$ & $\mathrm{~A}, \mathrm{~B}, \mathrm{P}$ & Mg VIII & $2 \mathrm{p}^{22} \mathrm{D}-3 \mathrm{~s}{ }^{2} \mathrm{P}^{\mathrm{o}}$ & & Nist & $\mathrm{N}, \mathrm{SBu}, \mathrm{SFu}, \mathrm{SMu}, \mathrm{R}$ \\
\hline 87.46 & A & $\mathrm{Ne}$ VII & $2 \mathrm{~s}^{2}{ }^{1} \mathrm{~S}-3 \mathrm{~s}{ }^{1} \mathrm{P}^{\mathrm{o}}$ & G & Nist & $\mathrm{N}$ \\
\hline 87.72 & $\mathrm{~A}$ & Mg VII & $2 \mathrm{p}^{2}{ }^{1} \mathrm{D}-3 \mathrm{~d}{ }^{1} \mathrm{D}^{\mathrm{o}}$ & M & Kelly & $\mathrm{N}$ \\
\hline $88.08,88.12$ & $\mathrm{~A}, \mathrm{~B}, \mathrm{P}$ & Ne VIII & $2 \mathrm{~s}^{2} \mathrm{~S}-3 \mathrm{p}^{2} \mathrm{P}^{\mathrm{o}}$ & M & Nist & SA, SB, SF, SM, SMH, D, R \\
\hline 88.68 & (A), B, P & Mg VII & $2 \mathrm{p}^{2}{ }^{1} \mathrm{~S}-3 \mathrm{~d}{ }^{1} \mathrm{P}^{\mathrm{o}}$ & M & Kelly & $\mathrm{N}, \mathrm{SMu}$ \\
\hline $89.64, .65$ & $\mathrm{~A},(\mathrm{P})$ & $\mathrm{Mg}$ VI & $2 \mathrm{p}^{32} \mathrm{P}^{\mathrm{o}}-4 \mathrm{~s}{ }^{2} \mathrm{P}$ & M & Kelly & $\mathrm{N}, \mathrm{SBu}$ \\
\hline 91.56 & $\mathrm{P}$ & $\mathrm{Ne}$ VII & $2 \mathrm{p}^{1} \mathrm{P}^{\mathrm{o}}-4 \mathrm{~s}^{1} \mathrm{~S}$ & & Kelly & $\mathrm{SMu}, \mathrm{R}$ \\
\hline $92.13, .32$ & $\mathrm{~A}, \mathrm{~B}, \mathrm{P}$ & Mg VIII & $2 \mathrm{p}^{2}{ }^{2} \mathrm{~S}-3 \mathrm{~s}{ }^{2} \mathrm{P}^{\mathrm{o}}$ & & NisT & $\mathrm{N}, \mathrm{SMu}, \mathrm{SBu}, \mathrm{R}$ \\
\hline 92.85 & $\mathrm{P}$ & $\mathrm{Ne}$ VII & $2 \mathrm{p}^{2}{ }^{1} \mathrm{~S}-4 \mathrm{~d}^{1} \mathrm{P}^{\mathrm{o}}$ & M & Kelly & $\mathrm{SMu}, \mathrm{R}$ \\
\hline (93.89), 94.07, .10, (.27) & $\mathrm{A}, \mathrm{B}, \mathrm{P}$ & Mg VIII & $\begin{array}{c}2 \mathrm{p}^{2} \mathrm{P}-3 \mathrm{~s}{ }^{2} \mathrm{P}^{\mathrm{o}} \\
-\end{array}$ & & Nist & $\begin{array}{l}\text { N, (SAu, SBu), SMu, blend with } \\
\text { Fe X } 94.012 \AA, \text { Mg VII } 94.04 \AA\end{array}$ \\
\hline $94.04,(.17, .24)$ & $\mathrm{A}, \mathrm{B}, \mathrm{P}$ & Mg VII & $\begin{array}{c}2 \mathrm{p}^{35} \mathrm{~S}^{\mathrm{o}}-3 \mathrm{~s}{ }^{5} \mathrm{P} \\
-\end{array}$ & M & Kelly & $\begin{array}{l}\mathrm{N}, \mathrm{SMu}, \mathrm{SFu}, \text { blend with Fe X } 94.012 \AA \\
\text { noted by R, and Mg VIII } 94.07 \AA\end{array}$ \\
\hline
\end{tabular}

(ii) CHIANTI database (Young et al. 2003) ${ }^{2}$;

(iii) Kelly Atomic Line Database ${ }^{3}$.

However, in order to assemble the complete model atoms, other sources were essential, too:

(iv) Opacity Project (OP, Seaton et al. 1994) TOPbase ${ }^{4}$;

\footnotetext{
${ }^{2}$ http://wwwsolar.nrl.navy.mil/chianti.html

3 http://cfa-www.harvard.edu/amdata/ampdata/kelly/ kelly.html

${ }^{4}$ http://legacy.gsfc.nasa.gov/topbase/home.html
}

(v) University of Kentucky Atomic Line List ${ }^{5}$.

\section{Comparison of $\mathrm{H} 1504+65$ with $\alpha$ Cen A, $\alpha$ Cen B, and Procyon}

We have performed a detailed comparison of the H 1504+65 photospheric absorption line spectrum with the coronal emission line spectra of $\alpha$ Cen A, $\alpha$ Cen B, and Procyon. We have also used the model spectrum of $\mathrm{H} 1504+65$ for this purpose. It turns out that not all lines predicted by the model, particularly

\footnotetext{
5 http://www.pa.uky.edu/ $\sim$ peter/atomic/
} 
Table 1. continued.

\begin{tabular}{|c|c|c|c|c|c|c|}
\hline$\lambda / \AA ̊(H 1504+65$ model $)$ & Seen in & Ion & Transition & & Source & Remark \\
\hline $94.26, .27, .30, .31, .36, .39$ & $\mathrm{~B}$ & $\mathrm{Ne}$ VII & $2 p{ }^{3} \mathrm{P}^{\mathrm{o}}-3 \mathrm{p}^{3} \mathrm{P}$ & M & Bashkin & $\mathrm{N}, \mathrm{SMu}$ \\
\hline $95.03, .04$ & $\mathrm{~B}$ & Mg VII & $2 \mathrm{p}^{3}{ }^{3} \mathrm{D}^{\mathrm{o}}-3 \mathrm{~s}{ }^{3} \mathrm{D}$ & & Kelly & $\mathrm{N}$ \\
\hline $95.26, .38, .42, .49, .56, .65$ & $(\mathrm{~A}, \mathrm{~B}, \mathrm{P})$ & Mg VII & $\begin{array}{c}2 \mathrm{p}^{3}{ }^{3} \mathrm{P}-3 \mathrm{~s}{ }^{3} \mathrm{P}^{\mathrm{o}} \\
-\end{array}$ & G & Kelly & $\begin{array}{l}\text { N, SBu, SFu, SMu, blend with Fe X } 95.338 \AA \\
\text { noted by R; Mg VI } 95.42 \AA\end{array}$ \\
\hline$(95.38, .42, .48)$ & $(\mathrm{A}, \mathrm{B}, \mathrm{P})$ & Mg VI & $\begin{array}{c}2 \mathrm{p}^{3}{ }^{4} \mathrm{~S}^{\mathrm{o}}-3 \mathrm{~d}{ }^{4} \mathrm{P} \\
-\end{array}$ & G & Kelly & $\begin{array}{l}\text { SBu, SFu, SMu, R, blend with } \\
\text { Mg vII } 95.26-.65 \AA\end{array}$ \\
\hline $95.75, .81, .89, .90, .91,96.0$ & $\mathrm{~A}, \mathrm{~B}, \mathrm{P}$ & $\mathrm{Ne}$ VII & $\begin{array}{c}2 p{ }^{3} \mathrm{P}^{\mathrm{o}}-3 \mathrm{p}^{3} \mathrm{D} \\
-\end{array}$ & M & Bashkin & $\begin{array}{l}\text { N, SMu, broad emission, blend with } \\
\text { Si VI } 96.02 \AA \text { A noted by R }\end{array}$ \\
\hline $96.08, .09$ & $(\mathrm{~A}, \mathrm{~B}, \mathrm{P})$ & Mg VI & $\begin{array}{c}2 \mathrm{p}^{32} \mathrm{P}^{\mathrm{o}}-3 \mathrm{~d} "{ }^{2} \mathrm{D} \\
-\end{array}$ & M & Kelly & $\begin{array}{l}\text { N, SBu, SMu, blend with Fe X } 96.12 \AA \\
\text { noted by R }\end{array}$ \\
\hline 97.50 & $\mathrm{~A}, \mathrm{~B}, \mathrm{P}$ & $\mathrm{Ne}$ VII & $2 \mathrm{~s}^{2}{ }^{1} \mathrm{~S}-3 \mathrm{p}^{1} \mathrm{P}^{\mathrm{o}}$ & $\mathrm{G}$ & Kelly & SM, SW, SMH, R \\
\hline $98.11, .26$ & $\mathrm{~A}, \mathrm{~B}, \mathrm{P}$ & Ne VIII & $2 p^{2} \mathrm{P}^{\mathrm{o}}-3 \mathrm{~d}^{2} \mathrm{D}$ & & Nist & $\mathrm{SB}, \mathrm{SM}, \mathrm{SMH}, \mathrm{SW}, \mathrm{D}, \mathrm{R}$ \\
\hline $98.50, .51$ & $\mathrm{~B}$ & Mg VI & $2 \mathrm{p}^{32} \mathrm{P}^{\mathrm{o}}-3 \mathrm{~d}{ }^{2} \mathrm{~S}$ & M & Kelly & $\mathrm{N}, \mathrm{SBu}, \mathrm{SMu}$ \\
\hline 99.69 & $\mathrm{~B}$ & OVI & $2 s-6 p$ & G & Kelly & $\mathrm{N}, \mathrm{SMu}$ \\
\hline $100.70, .90$ & A & Mg VI & $2 \mathrm{p}^{32} \mathrm{D}^{\mathrm{o}}-3 \mathrm{~d}^{2} \mathrm{~F}$ & M & Kelly & $\mathrm{N}, \mathrm{SBu}$ \\
\hline $101.49, .55$ & $\mathrm{~B}$ & $\mathrm{Mg}$ VI & $2 \mathrm{p}^{32} \mathrm{D}^{\mathrm{o}}-3 \mathrm{~d}^{2} \mathrm{P}$ & M & Kelly & $\mathrm{N}, \mathrm{SBu}, \mathrm{SMu}$ \\
\hline $102.91,103.08$ & $\mathrm{~A}, \mathrm{~B}, \mathrm{P}$ & Ne VIII & $2 p^{2} \mathrm{P}^{\mathrm{o}}-3 \mathrm{~s}^{2} \mathrm{~S}$ & & Nist & SM, SMH, SW, D, R \\
\hline 103.09 & $(\mathrm{~A}, \mathrm{~B}, \mathrm{P})$ & Ne VII & $2 p{ }^{1} P^{o}-3 p^{1} D$ & & Kelly & N, SBu, blend with Ne VIII $103.08 \AA$ \\
\hline 104.81 & $\mathrm{~B}, \mathrm{P}$ & O VI & $2 s-5 p$ & G & Kelly & $\mathrm{SMu}, \mathrm{R}$ \\
\hline 105.17 & $\mathrm{~A},(\mathrm{~B})$ & Mg VII & $2 \mathrm{p}^{3}{ }^{1} \mathrm{D}^{\mathrm{o}}-3 \mathrm{~s}^{1}{ }^{1} \mathrm{D}$ & & Kelly & N, SMu, blend with Fe IX $105.21 \AA$ noted by R \\
\hline $106.03, .08, .19$ & $\mathrm{P}$ & Ne VII & $2 p^{3} \mathrm{P}^{\mathrm{o}}-3 \mathrm{~d}^{3} \mathrm{D}$ & M & Kelly & N, SM, SW, D, R \\
\hline$(111.10, .16), .26$ & $\mathrm{~A}, \mathrm{~B}, \mathrm{P}$ & $\mathrm{Ne}$ VI & $\begin{array}{c}2 p^{2} \mathrm{P}^{\mathrm{o}}-3 \mathrm{p}^{2} \mathrm{D} \\
-\end{array}$ & G & Kelly & $\begin{array}{l}\mathrm{N}, \mathrm{SBu}, \mathrm{SMu} \text {, blend with } \mathrm{Ca} X 111.20 \\
\text { poss. Mg VI contribution noted by } \mathrm{R}\end{array}$ \\
\hline 111.15 & (A), B, P & $\mathrm{Ne}$ VII & $\begin{array}{c}2 \mathrm{p}^{2}{ }^{1} \mathrm{D}-3 \mathrm{~d}^{1} \mathrm{P}^{\mathrm{o}} \\
-\end{array}$ & M & Kelly & $\begin{array}{l}\mathrm{N} \text {, blend with Ca X } 111.20 \text {, Ne VI } 111.16 \AA \\
\text { poss. Mg VI contribution noted by } \mathrm{R}\end{array}$ \\
\hline $111.55, .75, .86$ & $\mathrm{~B},(\mathrm{~A}, \mathrm{P})$ & Mg VI & $2 \mathrm{p}^{3}{ }^{4} \mathrm{~S}^{\mathrm{o}}-3 \mathrm{~s}{ }^{4} \mathrm{P}$ & G & Kelly & $\mathrm{SB}, \mathrm{R}$ \\
\hline$(115.33), .39,(.52)$ & $\mathrm{A}, \mathrm{B}, \mathrm{P}$ & $\mathrm{Ne}$ VII & $2 p^{3} \mathrm{P}^{\mathrm{o}}-3 \mathrm{~s}^{3} \mathrm{~S}$ & M & Kelly & $\mathrm{R}$ \\
\hline $115.82, .83$ & $\mathrm{~B}$ & O VI & $2 s-4 p$ & $\mathrm{G}$ & Kelly & $\mathrm{SB}, \mathrm{SMu}, \mathrm{Ru}$ \\
\hline 115.96 & B & $\mathrm{Ne}$ VII & $2 \mathrm{p}^{2}{ }^{1} \mathrm{D}-3 \mathrm{~d}^{1} \mathrm{D}^{\mathrm{o}}$ & M & Kelly & $\mathrm{N}$ \\
\hline$(116.35), .42$ & B & O VI & $2 p-5 d$ & & Kelly & $\mathrm{N}$ \\
\hline 116.69 & B & $\mathrm{Ne}$ VII & $2 p{ }^{1} P^{o}-3 d^{1} D$ & & Kelly & $\mathrm{SMu}, \mathrm{R}$ \\
\hline $116.97,117.22$ & A & $\mathrm{Mg}$ VI & $2 \mathrm{p}^{32} \mathrm{D}^{\mathrm{o}}-3 \mathrm{~s}{ }^{2} \mathrm{P}$ & M & Kelly & $\mathrm{N}, \mathrm{SMu}$, poss. Mg VI contribution noted by $\mathrm{R}$ \\
\hline$(117.33), .40$ & $\mathrm{~B}$ & O VI & $2 p-5 s$ & & Kelly & $\mathrm{N}$ \\
\hline$(117.43), .66,(.78)$ & $\mathrm{P}$ & Mg VII & $2 \mathrm{p}^{3}{ }^{3} \mathrm{~S}^{\mathrm{o}}-3 \mathrm{~s}{ }^{3} \mathrm{P}$ & & Kelly & $\mathrm{N}, \mathrm{Ru}$ \\
\hline$(117.52), .64,(.81)$ & $\mathrm{P}$ & Mg VII & $2 \mathrm{p}^{3}{ }^{3} \mathrm{P}^{\mathrm{o}}-3 \mathrm{p}{ }^{3} \mathrm{P}$ & & Kelly & $\mathrm{N}, \mathrm{Ru}$ \\
\hline $120.20, .27, .33, .35, .42, .48$ & $\mathrm{P}$ & Ne VII & $2 \mathrm{p}^{2}{ }^{3} \mathrm{P}-3 \mathrm{~s}^{3} \mathrm{P}^{\mathrm{o}}$ & & Kelly & N, blend with O VII 120.33 Å note by R \\
\hline $122.49, .69$ & $\mathrm{~B}, \mathrm{P}$ & $\mathrm{Ne}$ VI & $2 \mathrm{p}^{2} \mathrm{P}^{\mathrm{o}}-3 \mathrm{~d}^{2} \mathrm{D}$ & G & Kelly & $\mathrm{N}, \mathrm{SBu}, \mathrm{SMu}, \mathrm{SMH}, \mathrm{D}, \mathrm{R}$ \\
\hline 123.59 & $\mathrm{P}$ & Mg VI & $2 \mathrm{p}^{42} \mathrm{D}-3 \mathrm{~s}^{\mathrm{iv} 2} \mathrm{D}^{\mathrm{o}}$ & & Kelly & $\mathrm{N}, \mathrm{SMu}, \mathrm{Ru}$ \\
\hline 127.67 & $\mathrm{~B}, \mathrm{P}$ & $\mathrm{Ne}$ VII & $2 \mathrm{p}{ }^{1} \mathrm{P}^{\mathrm{o}}-3 \mathrm{~s}{ }^{1} \mathrm{~S}$ & & Kelly & $\mathrm{SMu}, \mathrm{R}$ \\
\hline $129.78, .87$ & $\mathrm{~A}, \mathrm{~B}, \mathrm{P}$ & O VI & $2 p-4 d$ & & Kelly & SMH, SB, R \\
\hline $130.31, .64$ & $\mathrm{~B}$ & Mg VI & $2 \mathrm{p}^{42} \mathrm{P}-3 \mathrm{~s}^{\mathrm{v}} \mathrm{P}^{\mathrm{o}}$ & & Kelly & $\mathrm{N}$ \\
\hline $130.94,131.09, .30$ & $\mathrm{~A}, \mathrm{~B}, \mathrm{P}$ & Mg VII & $2 \mathrm{p}^{3} \mathrm{~S}^{\mathrm{o}}-3 \mathrm{p}{ }^{3} \mathrm{P}$ & & Kelly & $\begin{array}{l}\text { N, SBu, blend with Fe VIII 130.94, } 131.24 \AA \\
\text { noted by R }\end{array}$ \\
\hline $132.22, .31$ & A, B & O VI & $2 p-4 s$ & & Kelly & $\mathrm{N}$ \\
\hline $150.09, .12$ & $\mathrm{~B}, \mathrm{P}$ & O VI & $2 s-3 p$ & G & Kelly & SMH, SB, D, R \\
\hline
\end{tabular}

the weaker ones, are readily identified in $\mathrm{H} 1504+65$, which is at least in part due to the $\mathrm{S} / \mathrm{N}$ of the Chandra spectrum. Another reason is heavy blending by lines from iron group elements, which are not considered in the model used here. It was shown that identification of weak lines suffers from iron and nickel line blends, which is a problem because the accurate positions of the majority of lines from Fe-group elements in the soft X-ray domain is unknown (W04). The use of our synthetic spectrum in addition to the $\mathrm{H} 1504+65$ spectrum helps considerably to identify lines in the coronal spectra.

Table 1 summarizes the results of our comparison. Lines from 65 multiplets of $\mathrm{O}$ VI, Ne VI-VIII, and Mg VI-VIII are identified in both, $\mathrm{H} 1504+65$ (or its model) and in at least one of the considered coronae. Many of these had already been identified in earlier solar work (Widing \& Sandlin 1968; Freeman \& Jones 1970; Behring et al. 1972; Manson 1972; 


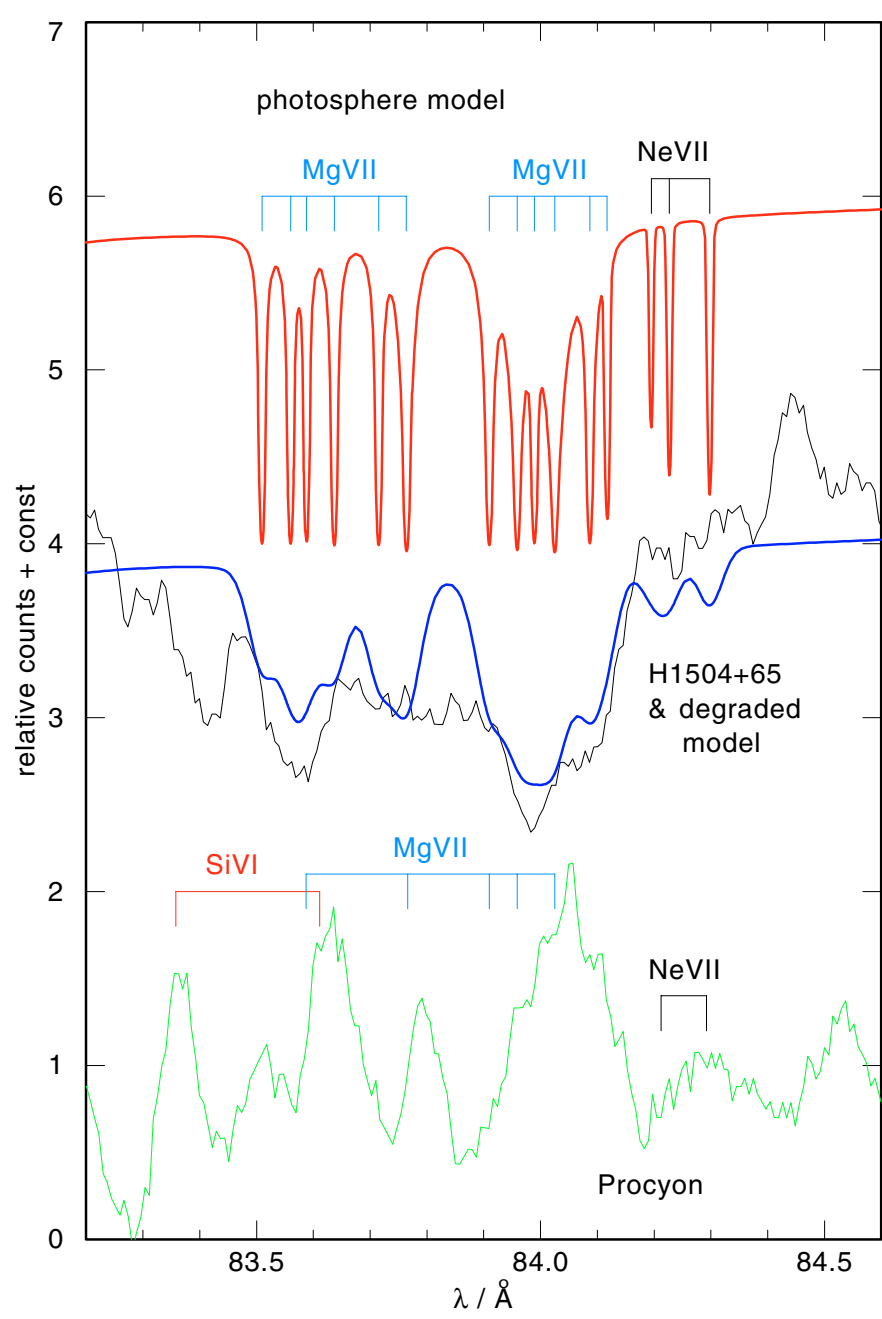

Fig. 1. Comparison of Chandra X-ray spectra of H1504+65 and Procyon. Lines from $\mathrm{Mg}$ VII and Ne VII are in absorption in H 1504+65 and in emission in Procyon. Top: photosphere model for H 1504+65 with line identifications for Mg VII and Ne VII. Middle: degraded model spectrum (i.e. folded with a $0.05 \AA F W H M$ Gaussian) plotted over H $1504+65$ observation. Bottom: Procyon spectrum with line identifications from Raassen et al. (2002). Chandra spectra were smoothed with a $0.03 \AA$ A boxcar.

Malinovsky \& Heroux 1973; Acton et al. 1985) and by Raassen et al. (2002, 2003), but the majority represents new identifications. Table 1 also denotes lines or features seen in earlier solar spectra but which were unidentified in the earlier work. The identifications presented here can then also be applied (either wholly or in part, allowing for blends) to these solar spectra. Many, but not all, of the tabulated lines have lower levels which are either ionic ground states or metastable states (labeled $\mathrm{G}$ or $\mathrm{M}$, respectively). As an example how the spectra compare, we show in Fig. 1 the spectra of Procyon and H 1504+65 in a wavelength region where a bunch of lines from two $\mathrm{Mg}$ VII and one Ne VII multiplet is located. All three multiplets, or at least some components of them, were identified by Raassen et al. (2002) in Procyon. They are also clearly seen as absorption features in the $\mathrm{H} 1504+65$ spectrum. Over this, we have plotted the model spectrum, degraded to the Chandra spectral resolution, which can qualitatively reproduce the observed line features. Placed at the top of this figure we show the original, non-degraded model spectrum, showing the diverse structure of the multiplets, whose components are not entirely resolved in Chandra spectra, neither of $\mathrm{H} \mathrm{1504+65}$ nor of Procyon.

Figure 2 shows a detail from the spectra of Procyon and $\alpha$ Cen A compared to $\mathrm{H} 1504+65$ in another wavelength interval. It displays some new line identifications in the coronal spectra, see for example the $87.46 \AA$ resonance line of $\mathrm{Ne}$ VII in $\alpha$ Cen A. The strongest emissions in $\alpha$ Cen A stem from two Ne VIII and Mg VIII doublets, identified already in Raassen et al. (2003). But note that the Mg VIII $86.84 \AA$ component is blended with the possibly stronger, newly identified Ne VII $86.82 \AA$ line.

Some of the newly identified lines do blend with other lines used for coronal diagnostics. The emissivity of the Fe VIII lines at $130.94 \AA$ and $132.24 \AA$ in Procyon was computed by Raassen et al. (2002) using a three-temperature model. They stress that these line strengths are strongly underestimated, by factors 6 and 4 compared to the observation. The result of their differential emission measure (DEM) model underestimates the emissivity even more (factors 9 and 6). This can at least partially be explained by the fact that two components of a Mg VII triplet (at $130.94 \AA$ and $131.30 \AA$ ) can contribute to the Fe VIII line emissivities. A similar explanation may hold for the Fe IX 105.20 $\AA$ line, which also appeared too weak in their model. It is blended with a Mg VII singlet at $105.17 \AA$.

Another example is the Mg VIII $74.86 \AA$ line observed in $\alpha$ Cen A and $\alpha$ Cen B. Raassen et al. (2003) find that the line fluxes from their models are too small by about $40 \%$. We think that the missing flux is contributed by a blend with a new neon line located at almost the same wavelength, Ne VII $74.87 \AA$. Detailed emission measure modeling, which is beyond the scope of this paper, is needed to quantify these suggestions. Other blends with previously identified emission lines in the coronae of Procyon and $\alpha$ Cen are indicated in Table 1.

\section{Summary}

We have performed a detailed comparison of Chandra soft $\mathrm{X}$-ray spectra from the photosphere of the hottest known white dwarf, H 1504+65, with the corona spectra of $\alpha$ Cen A, $\alpha$ Cen B, and Procyon. With the help of a detailed model spectrum for $\mathrm{H} 1504+65$ we have found that a large number of lines from multiplets of $\mathrm{O}, \mathrm{Ne}$, and $\mathrm{Mg}$ are present in both the photospheric absorption line spectrum and the coronal emission line spectra. In the coronal spectra we have newly identified lines from about 40 multiplets of O VI, Ne VI-VII, and Mg VI-VIII. Some of these lines are blends with previously known lines, which are in use for diagnostic purposes, hence, their contribution to the line flux must be considered in detailed spectral analyses.

Acknowledgements. Analysis of X-ray data in Tübingen is supported by the DLR under grant 50 OR 0201. J.J.D. was supported by NASA contract NAS8-39073 to the Chandra X-ray Center. 


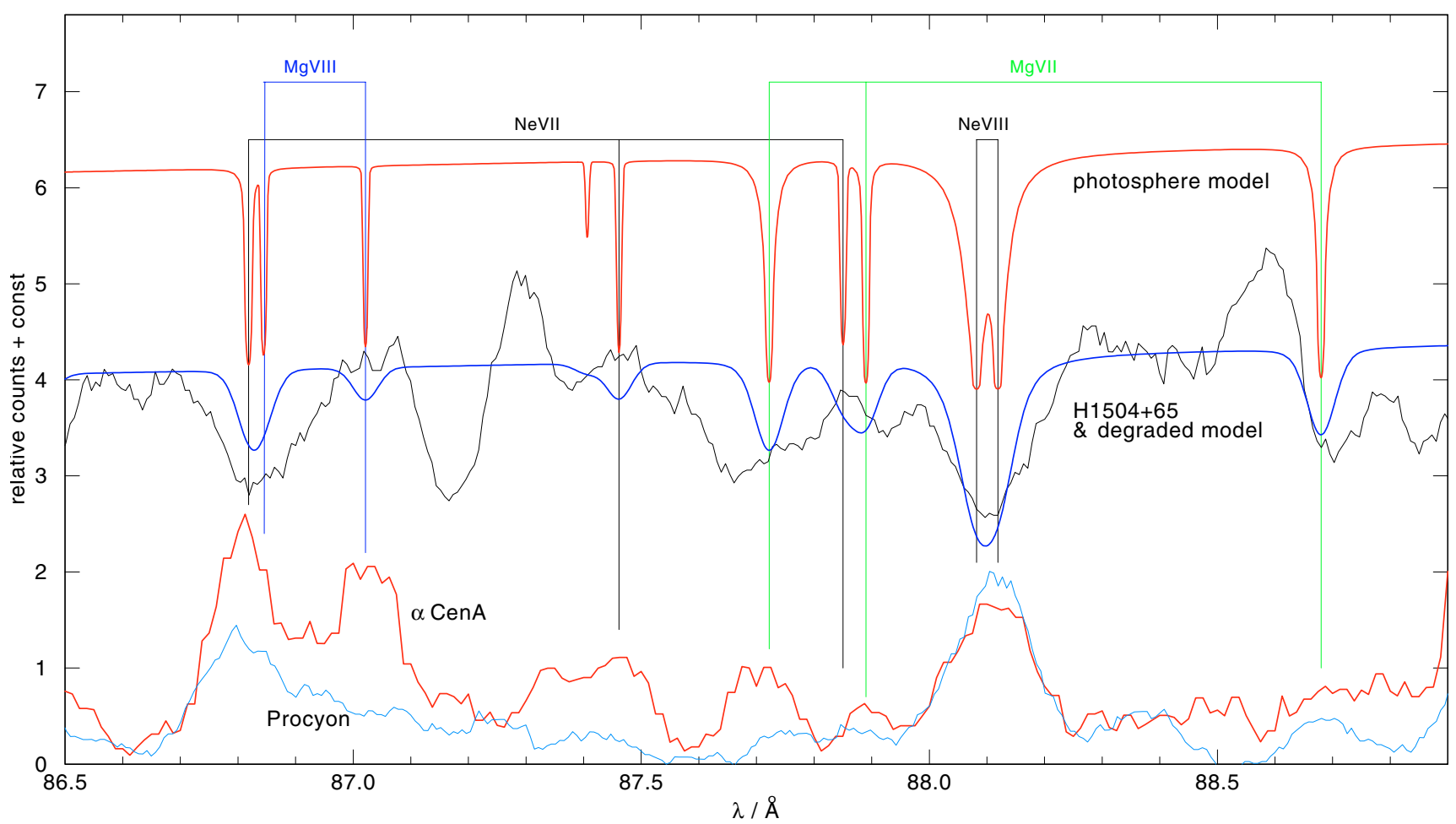

Fig. 2. Comparison of Chandra X-ray spectra of $\mathrm{H} 1504+65$ with Procyon and $\alpha$ Cen A, similar to Fig. 1. All shown lines from highly ionized $\mathrm{Ne}$ and $\mathrm{Mg}$ are identified for the first time in the cool star corona, except for Mg VIII 86.85/87.02 $\AA$ and the Ne VIII doublet at 88.1 $\AA$, which were identified by Raassen et al. (2002, 2003). Chandra spectra of H $1504+65$ and the coronae were smoothed with $0.03 \AA$ and $0.05 \AA$ boxcars, respectively.

\section{References}

Acton, L. W., Bruner, M. E., Brown, W. A., et al. 1985, ApJ, 291, 865 Behring, W. E., Cohen, L., \& Feldman, U. 1972, ApJ, 175, 493

Beiersdorfer, P., Lepson, J. K., Brown, G. V., et al. 1999, ApJ, 519, L185

Drake, J. J. 1996, in Cool Stars, Stellar Systems; and the Sun: 9, ed. R. Pallavicini, A. K. Dupree, ASP Conf. Ser., 109, 203

Drake, J. J., Laming, J. M., \& Widing, K. G. 1995, ApJ, 443, 393

Drake, J. J., Laming, J. M., \& Widing, K. G. 1997, ApJ, 478, 403

Freeman, F. F., \& Jones, B. B. 1970, Sol. Phys., 15, 288

Jordan, C. 1996, in Astrophysics in the Extreme Ultraviolet, ed. S. Bowyer, R.F. Malina (Dordrecht: Kluwer Academic Publ.), IAU Coll. 152, 81

Lepson, J. K., Beiersdorfer, P., Brown, G. V., et al. 2002, ApJ, 578, 648
Lepson, J. K., Beiersdorfer, P., Behar, E., \& Kahn, S. M. 2003, ApJ, 590,604

Malinovsky, L., \& Heroux, M. 1973, ApJ, 181, 1009

Manson, J. E. 1972, Sol. Phys., 27, 107

Raassen, A. J. J., Mewe, R., Audard, M., et al. 2002, A\&A, 389, 228

Raassen, A. J. J., Ness, J.-U., Mewe, R., et al. 2003, A\&A, 400, 671

Seaton, M. J., Yan, Y., Mihalas, D., \& Pradhan, A. K. 1994, MNRAS, 266, 805

Schweizer, W., \& Schmidtke, G. 1971, ApJ, 169, L27

Werner, K. 2001, in Low Mass Wolf-Rayet Stars: Origin and Evolution, ed. T. Blöcker, L. B. F. M. Waters, A. A.Zijlstra, Ap\&SS, 275, 27

Werner, K., Rauch, T., Barstow, M. A., \& Kruk, J. W. 2004, A\&A, 421, 1169

Widing, K. G., \& Sandlin, G. D. 1968, ApJ, 152, 545

Young, P. R., Del Zanna, G., Landi, E., et al. 2003, ApJS, 144, 135 